\title{
Weierstrass' Elliptic Function Solution to the Autonomous Limit of the String Equation of Type $(2,5)^{*}$
}

\author{
Yoshikatsu Sasaki \\ Department of Mathematics, Hiroshima University, Higashi-Hiroshima, Japan \\ Email: sasakiyo@hiroshima-u.ac.jp
}

Received 1 June 2014; revised 3 July 2014; accepted 15 July 2014

Copyright (C) 2014 by author and Scientific Research Publishing Inc.

This work is licensed under the Creative Commons Attribution International License (CC BY).

http://creativecommons.org/licenses/by/4.0/

cc) (i) Open Access

\begin{abstract}
In this article, we study the string equation of type $(2,5)$, which is derived from $2 \mathrm{D}$ gravity theory or the string theory. We consider the equation as a 4 th order analogue of the first Painlevé equation, take the autonomous limit, and solve it concretely by use of the Weierstrass' elliptic function.
\end{abstract}

\section{Keywords}

\section{Painlevé Hierarchy, String Equation, Elliptic Function}

\section{Introduction}

\subsection{The String Equation of Type $(2,5)$}

Put $D=\mathrm{d} / \mathrm{d} z$. Consider the commutator equation of ordinary differential operators

$$
[Q, P]=1, \quad Q:=\Sigma_{k=2}^{q} w_{k} D^{q-k}, \quad P:=\sum_{k=2}^{p} v_{k} D^{p-k} .
$$

We call it the string equation (or Douglas equation) of type $(q, p)$, which appears in the string theory or the theory of quantum gravity in 2D [1]-[9]. In the followings, we set $q=2, p=2 g+1$.

In the case where $q=2, p=3$, the string equation is written as an ODE satisfied by the potential $w$ of Sturm-Liouville operator $Q=D^{2}+w$, and then, by a fractional linear transformation, it is reduced to the first Painlevé equation [10] [11]

$$
w^{\prime \prime}=6 w^{2}+z,
$$

"Dedicated to Professor Masafumi Yoshino on the occasion of his 60th birthday. 
which is equivalent to the Hamiltonian system:

$$
\mathrm{d} w / \mathrm{d} z=\partial H / \partial v, \quad \mathrm{~d} v / \mathrm{d} z=-\partial H / \partial w, \quad H=\frac{1}{2} v^{2}-2 w^{3}-z w .
$$

In the case where $q=2, p=5,[Q, P]=1$ yields

$$
C_{0}=w^{(4)}+5 w^{\prime 2}+10\left(w^{\prime}+C_{1}\right)\left(w^{\prime \prime}+3 w^{2}\right)-20 w^{3}+16 C_{2} w+16 z,
$$

where $C_{0}, C_{1}, C_{2}$ are integral constants. By the fractional linear transformation $z \mapsto \alpha z+\beta, w \mapsto \gamma w+\delta$,

$$
\alpha^{7}=-\frac{1}{3}, \quad \gamma=6 \alpha^{5}, \quad \delta=-C_{1}, \quad 16 \beta=C_{0}-20 C_{1}^{3}+16 C_{1} C_{2}
$$

and putting $a=\alpha^{4}\left(8 C_{2}-15 C_{1}^{2}\right) / 4$, the string equation is reduced to

$$
w^{(4)}=20 w^{\prime \prime} w+10 w^{\prime 2}-40 w^{3}-8 a w-\frac{8}{3} z,
$$

We also call it the string equation of type $(2,5)$. Note that (S) coincides the 4th order equation of the first Painlevé hierarchy [12]-[15]

$$
d_{n}[w]+4 z=0,
$$

for $n \in \mathbb{N}$, where $d_{n}[w]$ is an expression of a given meromorphic function $w$ defined by $d_{0}[w]=-4 w$ and $D d_{n+1}[w]=\left(D^{3}-8 w D-4 w^{\prime}\right) d_{n}[w]$.

\subsection{Degenerated Garnier System}

Equation (S) is also obtained as follows. Consider a 2D degenerated Garnier system [16] [17]:

$$
\begin{gathered}
\partial q_{i} / \partial t_{j}=\partial H_{j} / \partial p_{i}, \quad \partial p_{i} / \partial t_{j}=-\partial H_{j} / \partial q_{i}, \quad(i, j \in\{1,2\}), \\
H_{1}=\frac{1}{3}\left(q_{2}^{2}-q_{1}-\frac{1}{3} t_{1}\right) p_{1}^{2}+\frac{2}{3} q_{2} p_{1} p_{2}+\frac{1}{3} p_{2}^{2}+3\left(q_{1}+\frac{1}{3} t_{1}\right) q_{2}\left(q_{2}^{2}-2 q_{1}+\frac{1}{3} t_{1}\right)-t_{2} q_{1}, \\
H_{2}=\frac{1}{3} q_{2} p_{1}^{2}+\frac{2}{3} p_{1} p_{2}+3 q_{2}^{4}-9 q_{1} q_{2}^{2}+3 q_{1}^{2}-t_{1} q_{1}-t_{2} q_{2} .
\end{gathered}
$$

which is a $2 \mathrm{D}$ analogue of (PI) in the theory of isomonodromic deformations. If we fix one of the independent variables $t_{1} \equiv a$ (= const. $)$, we get a Hamiltonian system with only one independent variable $t_{2} \equiv z$ as follows:

$$
\begin{aligned}
& \partial q_{i} / \partial z=\partial H / \partial p_{i}, \quad \partial p_{i} / \partial z=-\partial H / \partial q_{i}, \quad(i \in\{1,2\}), \\
& H\left(=H_{2}\right)=\frac{1}{3} q_{2} p_{1}^{2}+\frac{2}{3} p_{1} p_{2}+3 q_{2}^{4}-9 q_{1} q_{2}^{2}+3 q_{1}^{2}-a q_{1}-z q_{2} .
\end{aligned}
$$

From the above system, eliminating $q_{1}, p_{1}, p_{2}$ and putting $w=q_{2}$, we obtain (S). So, Equation (S) is 4 th order analogue of (PI) in the double sences.

It is already known by Shimomura [18] that every solution to (S) is meromorphic on $\mathbb{C}$, and that every pole of every solution is double one with its residue 0 .

\subsection{Autonomous Limit of the First Painlevé Equation}

The first Painlevé equation (PI) has the autonomous limit [11]. Replacing ( $w, v, z, H)$ by $\left(\varepsilon^{-2} w, \varepsilon^{-3} v, \varepsilon z+\varepsilon^{-4} b, \varepsilon^{-6} H\right)$ with a constant $b \in \mathbb{C}$, and taking limit $\varepsilon \rightarrow 0$, we obtain $w^{\prime \prime}=6 w^{2}+b$ which is solved by the Weierstrass' elliptic function [10] [11]. The relation between the fundamental 2-form before and after the replacement is

$$
\mathrm{d} w \wedge \mathrm{d} v-\mathrm{d} H \wedge \mathrm{d} z \mapsto \varepsilon^{-5}(\mathrm{~d} w \wedge \mathrm{d} v-\mathrm{d} H \wedge \mathrm{d} z)
$$

\subsection{Results}

It is quite natural to think that: 
Conjecture. Each equation of the first Painlevé hierarchy has the autonomous limit, and which is satisfied by the Weierstrass' elliptic function.

For $n=2$, the statement is valid, i.e.

Theorem A. Replacing $(w, z, a)$ by $\left(\varepsilon^{-2} w, \varepsilon z+\varepsilon^{-6} b, \varepsilon^{-4} a\right)$, or replacing $\left(q_{1}, q_{2}, p_{1}, p_{2}, z, H\right)$ by $\left(\varepsilon^{-4} q_{1}, \varepsilon^{-2} q_{2}, \varepsilon^{-3} p_{1}, \varepsilon^{-5} p_{2}, \varepsilon z+\varepsilon^{-6} b, \varepsilon^{-8} H\right)$ with a constant $b \in \mathbb{C}$, and taking limit $\varepsilon \rightarrow 0$, we obtain the autonomous limit of the 4th order equation of the first Painlevé hierarchy (S). Moreover, the relation between the fundamental 2-form before and after the replacement is

$$
\mathrm{d} p_{1} \wedge \mathrm{d} q_{1}+\mathrm{d} p_{2} \wedge \mathrm{d} q_{2}-\mathrm{d} H \wedge \mathrm{d} z \mapsto \varepsilon^{-7}\left(\mathrm{~d} p_{1} \wedge \mathrm{d} q_{1}+\mathrm{d} p_{2} \wedge \mathrm{d} q_{2}-\mathrm{d} H \wedge \mathrm{d} z\right)
$$

It is easy to show the above. The autonomous limit is given by

$$
w^{(4)}=20 w^{\prime \prime} w+10 w^{\prime 2}-40 w^{3}-8 a w-\frac{8}{3} b,
$$

Theorem B. The autonomous limit Equation (A) has a solution concretely described by the Weierstrass' elliptic function as

$$
w(z)=4 \wp(z) / a_{1}
$$

where $a_{1}=(8 \pm 4 \sqrt{-2}) / 3$.

Remark. Modulus of the elliptic function is determined by the constants $a$ and $b . g_{2}$ and $g_{3}$ in the elliptic function theory are as follows:

$$
g_{2}=-4 a_{1} a /\left(40-9 a_{1}\right), g_{3}=-a_{1}^{2} b / 6\left(10-3 a_{1}\right) .
$$

The next section is devoted to give the proof of Theorem $\mathrm{B}$.

\section{Proof of Theorem B}

Put $\varphi=-[$ l.h.s. of $(\mathrm{A})]+[$ r.h.s. of $(\mathrm{A})]$, i.e.

$$
\varphi:=w^{(4)}+20 w^{\prime \prime} w+10 w^{\prime 2}-40 w^{3}-8 a w-\frac{8}{3} b=0 .
$$

Multiplying both sides of $\varphi=0$ by $w^{\prime}$, and integrating it, we obtain a first integral of (A)

$$
\int \varphi w^{\prime} \mathrm{d} z:=-w^{\prime} w^{\prime \prime \prime}+\frac{1}{2} w^{\prime \prime 2}+10 w^{\prime 2} w-10 w^{4}-4 a w^{2}-\frac{8}{3} b w=c: \text { const. }
$$

In order to find the elliptic function solution, let $w$ satisfy the relation:

$$
w^{\prime 2}=a_{0} w^{4}+a_{1} w^{3}+a_{2} w^{2}+a_{3} w+a_{4}=: A(w) .
$$

Substituting (3), $w^{\prime \prime}=\frac{1}{2} A_{w}(w)$ and $w^{\prime \prime \prime}=\frac{1}{2} A_{w w}(w) w^{\prime}$ into (2), we have

$$
\begin{aligned}
\int \varphi w^{\prime} \mathrm{d} z= & -\frac{1}{2} A_{w w}(w) A(w)+\frac{1}{8} A_{w}(w)^{2}+10 w A(w)-10 w^{4}-4 a w^{2}-\frac{8}{3} b w=c \\
= & {\left[-4 a_{0}^{2}\right] w^{6}+\left[-6 a_{0} a_{1}+10 a_{0}\right] w^{5}+\left[-5 a_{0} a_{2}-\frac{15}{8} a_{1}^{2}+10 a_{1}-10\right] w^{4} } \\
& +\left[-5 a_{0} a_{3}-\frac{5}{2} a_{1} a_{2}+10 a_{2}\right] w^{3}+\left[-6 a_{0} a_{4}-\frac{9}{4} a_{1} a_{3}-\frac{1}{2} a_{2}^{2}+10 a_{3}-4 a\right] w^{2} \\
& +\left[-3 a_{1} a_{4}-\frac{1}{2} a_{2} a_{3}+10 a_{4}-\frac{8}{3} b\right] w+\left[-a_{2} a_{4}+\frac{1}{8} a_{3}^{2}\right] \cdot 1 .
\end{aligned}
$$

So, if we take

$$
a_{0}=a_{2}=0, a_{1}=\frac{1}{3}(8 \pm 4 \sqrt{-2}), a_{3}=16 a /\left(40-9 a_{1}\right), a_{4}=8 b / 3\left(10-3 a_{1}\right) \text {, and } c=\frac{1}{8} a_{3}^{2} \text {, }
$$

then solutions of (3) satisfy (2). Now, in order to reduce $w^{\prime 2}=a_{1} w^{3}+a_{3} w+a_{4}$ to $\wp^{\prime 2}=4 \wp^{3}-g_{2} \wp-g_{3}$, we 
use the scale transformation $w=\chi \wp, \chi \in \mathbb{C} \backslash\{0\}$. Immediately we obtain $\chi=4 / a_{1}$, and also $g_{2}=-a_{3} / \chi$, $g_{3}=-a_{4} / \chi$.

\section{References}

[1] Douglas, M.R. (1990) String in Less than One-Dimensions and K-dV Hierarchies. Physics Letters B, 238, $176-180$. http://dx.doi.org/10.1016/0370-2693(90)91716-O

[2] Moore, G. (1990) Geometry of the String Equations. Communications in Mathematical Physics, 133, 261-304. http://dx.doi.org/10.1007/BF02097368

[3] Moore, G. (1991) Matrix Models of 2D Gravity and Isomonodromic Deformations. Progress of Theoretical Physics Supplement, 102, 255-285. http://dx.doi.org/10.1143/PTPS.102.255

[4] Fukuma, M., Kawai, H. and Nakayama, R. (1991) Infinite Dimensional Grassmannian Structure of Two Dimensional String Theory. Communications in Mathematical Physics, 143, 371-403. http://dx.doi.org/10.1007/BF02099014

[5] Kac, V. and Schwarz, A. (1991) Geometric Interpretation of Partition Functions of 2D Gravity. Physics Letters B, 257, 329-334. http://dx.doi.org/10.1016/0370-2693(91)91901-7

[6] Schwarz, A. (1991) On Solutions to the String Equations. Modern Physics Letters A, 29, 2713-2725. http://dx.doi.org/10.1142/S0217732391003171

[7] Adler, M. and van Moerbeke, P. (1992) A Matrix Integral Solution to Two-Dimensional $W_{p}$-Gravity. Communications in Mathematical Physics, 147, 25-26. http://dx.doi.org/10.1007/BF02099527

[8] van Moerbeke, P. (1994) Integrable Foudations of String Theory. In: Babelon, O., et al., Ed., Lectures on Integrable Systems, World Science Publisher, Singapore, 163-267.

[9] Takasaki, K. (2007) Hamiltonian Structure of PI Hierarchy. SIGMA, 3, 42-116.

[10] Ince, E.L. (1956) Ordinary Differential Equations. Dover Publications, New York.

[11] Conte, R. and Mussette, M. (2008) The Painlevé Handbook. Springer Science + Business Media B.V., Dordrecht.

[12] Weiss, J. (1984) On Classes of Integrable Systems and the Painlevé Property. Journal of Mathematical Physics, 25, 13-24. http://dx.doi.org/10.1063/1.526009

[13] Kudryashov, N.A. (1997) The First and Second Painlevé Equations of Higher Order and Some Relations between Them. Physics Letters A, 224, 353-360. http://dx.doi.org/10.1016/S0375-9601(96)00795-5

[14] Gromak, V.I., Laine, I. and Shimomura, S. (2002) Painlevé Differential Equations in the Complex Plane. Walter de Gruyter, Berlin. http://dx.doi.org/10.1515/9783110198096

[15] Shimomura, S. (2004) Poles and $\alpha$-Points of Meromorphic Solutions of the First Painlevé Hierarchy. Publications of the Research Institute for Mathematical Sciences, Kyoto University, 40, 471-485. http://dx.doi.org/10.2977/prims/1145475811

[16] Kimura, H. (1989) The Degeneration of the Two Dimensional Garnier System and the Polynomial Hamiltonian Structure. Annali di Matematica Pura ed Applicata, 155, 25-74. http://dx.doi.org/10.1007/BF01765933

[17] Suzuki, M. (2006) Spaces of Initial Conditions of Garnier System and Its Degenerate Systems in Two Variables. Journal of the Mathematical Society of Japan, 58, 1079-1117. http://dx.doi.org/10.2969/jmsj/1179759538

[18] Shimomura, S. (2000) Painlevé Property of a Degenerate Garnier System of (9/2)-Type and a Certain Fourth Order Non-Linear Ordinary Differential Equation. Annali della Scuola Normale Superiore di Pisa, Classe di Scienze, 29, $1-17$. 
Scientific Research Publishing (SCIRP) is one of the largest Open Access journal publishers. It is currently publishing more than 200 open access, online, peer-reviewed journals covering a wide range of academic disciplines. SCIRP serves the worldwide academic communities and contributes to the progress and application of science with its publication.

Other selected journals from SCIRP are listed as below. Submit your manuscript to us via either submit@scirp.org or Online Submission Portal.
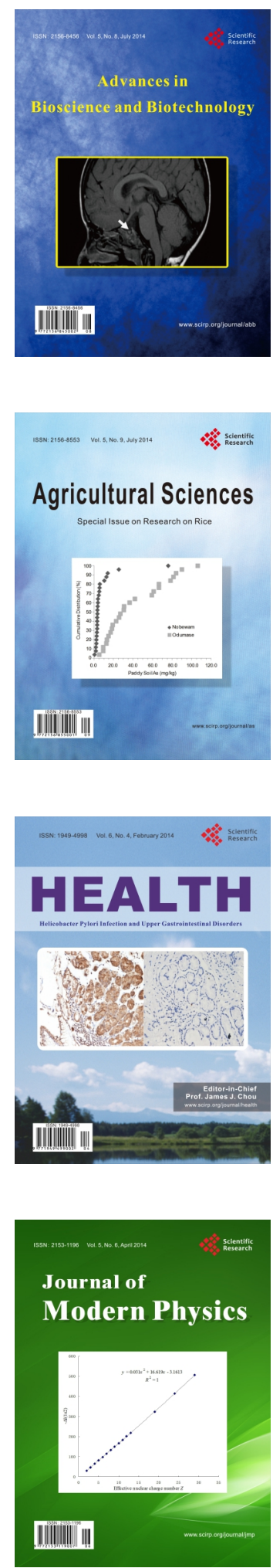
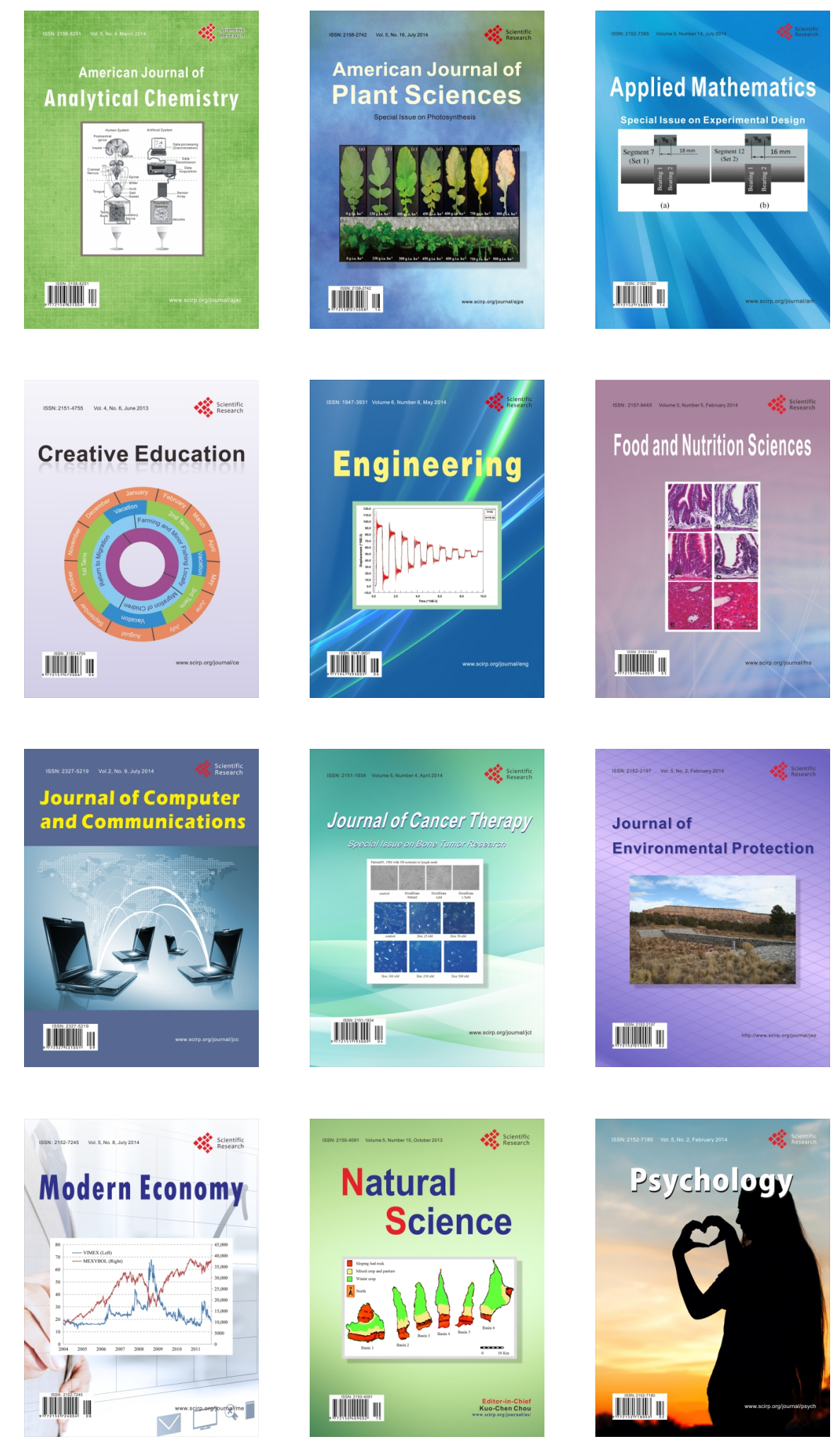